\title{
Zero energy buildings in the logistics warehouse systems
}

\author{
Pawel Zajac ${ }^{1, *}$, Stanislaw Kwasniowski ${ }^{1}$ \\ ${ }^{1}$ Wroclaw University of Science and Technology, Faculty of Mechanical Engineering, Wybrzeze \\ Wyspianskiego 27, 50-370 Wroclaw, Poland
}

\begin{abstract}
The article discusses the evaluation of possible reductions of energy consumption in warehouse buildings and the analysis of construction and functioning of modern storage warehouses. For a warehouse the following are presented: operation evaluation measures and indexes, an energy balance, ideas for improvements in terms of energy conservation in particular functioning zones.
\end{abstract}

\section{Introduction}

The functioning of warehouses is clearly related to energy consumption in its various forms. Among various forms and varieties of warehouses, the cold and heated warehouses are characterized by relatively high energy demand. Modern warehouse buildings often cover tens of thousands square meters. The energy demand in such types of buildings is enormous. Not only is the economic aspect essential, but also ecological one. The renewable energy sources support natural environment protection. Investments in such types of sources are often funded. Therefore if we assume that a zero energy building is a building that does not consume energy in an annual balance, then logistic warehouse systems seek just such solutions [1].

\section{Warehouse energy balance}

Many goods require controlled storage conditions. Therefore, warehouse buildings are equipped with devices that enable creating a suitable microclimate inside. Thus these will be cold, air conditioned and heated warehouses [2].

Depending on intended use, cold warehouses may require an internal temperature of even $-40^{\circ} \mathrm{C}$. The air-conditioned warehouses are the warehouses in which normal conditions are maintained. The heated warehouses are buildings in which the temperature should be above zero $T_{w}>0^{\circ} \mathrm{C}$. Obviously there are special warehouses that require maintaining higher temperatures [3].

The present analysis considers typical warehouse buildings in which various goods are stored, equipment is operated and people work. In such a situation we treat warehouse buildings as facilities in which interior temperature is practically constant and its little

\footnotetext{
*Corresponding author: pawel.zajac@pwr.edu.pl
} 
fluctuations resulting from the working control systems are ignored. External and internal factors determine the condition of the warehouse interior and the goods stored within. Two situations can be distinguished. Case I - a warehouse requires energy supply. Case II a warehouse requires draining energy. These situations are described by the heat balance equation.

$$
Q_{d o p} /_{\text {odp }}=Q_{\text {przen }}+Q_{w e w}+Q_{u r z}+Q_{o s ́ w}+Q_{w e w . w e n}+Q_{o s}-\Delta E_{p}
$$

Symbols in the equation (1) represent: $Q_{d o p^{-}}$heat supplied by warehouse heating system; $Q_{o d p}-$ heat removed from interior by interior cooling equipment; $\boldsymbol{Q}_{\boldsymbol{p}}-$ heat penetrating through insulated warehouse walls with different thermal insulation of walls, windows, roof, floor particular areas taking also into account thermal bridges. The flux presents the equation (2) [4].

$$
Q_{p r z e n}=Q_{p n n}+Q_{p o s}+Q_{p n}+Q_{o k}+Q_{o k n}+Q_{p b p}+Q_{m}
$$

Symbols in the equation (2) represent: $Q_{\text {przen }}$ - heat of penetration; $Q_{p n n}-$ heat of penetration through sunless walls; $Q_{p o s}-$ heat of penetration through floor; $Q_{p n}$ - heat of penetration through sunlit walls; $Q_{o k}-$ heat of penetration through windows; $Q_{o k n}-$ heat of penetration through windows or skylights in radiation path; $Q_{p b p}$ - heat of penetration through walls from buildings adjoining to warehouse, e.g.: common-office rooms; $Q_{m}$ - heat of penetration through thermal bridges. The situation is presented in Fig. 1.

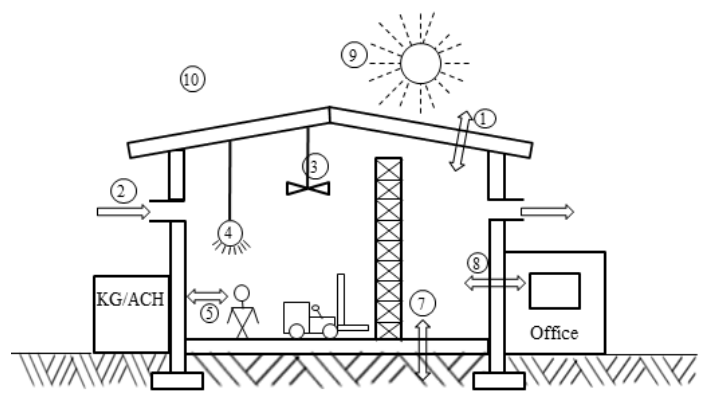

Fig. 1. Diagram of warehouse facility with internal and external energy sources: 1 - heat transfer through walls, 2 - facility ventilation, 3 - warehouse internal air supply devices, 4 - lighting, 5 - energy emitted by persons, 6 - energy emitted by devices, 7 - energy exchangeable with substrate (ground), 8 - energy exchangeable with adjoining buildings of different temperature, 9 - solar radiation energy, 10 - environment, 11 - heating boiler or refrigerating unit, 12 - office.

$$
Q_{p n n}=\left(T_{o t}-T_{w}\right) \sum k_{i} \cdot F_{i}
$$

Symbols in the equation (3) represent: $T_{o t}-$ ambient temperature; $T_{w}-$ interior temperature; $k_{i}$ - penetrability of given partition; $F_{i}$ - area of given partition. The value of heat losses through the so-called thermal bridges, depending on the warehouse structure, is given as a percentage part in relation to the total penetration heat losses. The heat penetration losses $Q_{p n n}$ are attempted to be reduced by using of suitable heat-insulating materials. It is worth to pay attention to the parameter of the heat penetration coefficient $k_{i}$ that expresses the Peclet dependence (4).

$$
k_{i}=\frac{1}{\frac{1}{\alpha_{z}}+\sum \frac{s_{i}}{\lambda_{i}}+\frac{1}{\alpha_{w}}}
$$


Symbols in the equation (8) represent: $\alpha_{z}$ - convective heat-transfer coefficient on external wall surface dependent on air flow around speed and surface condition (wet-dry); $\alpha_{w}$ - convective heat-transfer coefficient on internal wall surface depends on air flow around speed relative to wall; $s_{i}-$ thickness of distinguished partition; $\lambda_{i}-$ thermal conductivity of wall making layer. In case of differentiated walls construction, the heat balance takes into account the area and penetrability of each wall fragment of the same transverse structure. The analysis of convective heat-transfer coefficients $\frac{\alpha_{z}}{\alpha_{w}}$ impact on the " $\mathrm{k}$ " heat-transfer coefficient $\mathrm{k}$ is limited $(<1 \%)$ - here decides the wall thermal resistance $\frac{s_{i}}{\lambda_{i}}$ that is considerably higher than the thermal resistances on the building's external and internal surfaces.

$Q_{m}$ - heat penetrating through thermal bridges is estimated by the coefficients $Q_{p}$ - in vehicles it is assumed as equal to $10 \%$ of the penetration heat. In warehouse buildings it is proposed to assume $5 \% Q_{p}$. In the structure the so-called thermal bridges across the wall section are avoided. The thermal bridges can be: ventilating pipes, reinforcement bars, metal connections across wall, various types of connectors, metal door frames, levelling ramp metal platforms.

In many cases it is difficult to avoid such elements. Approx. 1000 times more heat runs away through a steel thermal bridge than through a heat-insulating wall of similar section. In practice it is easy to detect thermal bridges on the basis of thermovision tests.

Designers of warehouse buildings developed such solutions of constructional nodes that reduce the thermal bridge effects to a minimum.

$Q_{p o s}$ heat exchange through a floor of cold warehouse. The heat exchange through the floor can lead to ground freeze-up on which it is laid, which can result, in some circumstances, cracking of the floor and foundation (defrosting of frozen ground). Therefore the floor should be resistant to loads and, on the other hand, it should be well insulated so that not to lead to ground frost under it. In such a case it can be necessary to heat up the whole area of the floor. The waste heat of the condenser of the units cooling the warehouse room can be used to heat the floor. The heat flux supplied by the floor heating system, in accordance with the heat exchange theory, will be divided into two fluxes inversely proportional to the thermal resistance $\frac{s_{i}}{\lambda_{i}}$ of the insulation over the heating layer and under the heating layer. These layers are separated by a heated pipe screen (underfloor heating). The power of the heating system $Q_{p o s}$ will result from the thermal resistance of the insulation between the heating layer and the warehouse interior $k_{p}$ as well as the assumed temperature difference [5-7]:

$$
Q_{\text {pos }}=F_{p} \cdot k_{p}\left(T_{w y}-T_{w}\right)
$$

Symbols in the equation (5) represent: $F_{p}$ - floor area; $k_{p}$ - heat penetration of floor with insulation; $T_{w y}$ - heated layer temperature, screen with heating pipes; $T_{w}$ - warehouse interior temperature.

$\underline{Q}_{p n}$ - heat penetrating sunlit walls. The flux can be determined on the basis of heat radiation intensity, the area, the absorption coefficient and the walls heat-insulating features or from the equation of heat exchange through penetration, determining earlier the average calculation temperature of heat exchange.

$$
T_{o b l}=T_{o t}+\frac{A \cdot I}{\alpha_{z}}
$$


Symbols in the equation (6) represent: $\mathrm{A}-$ absorption coefficient depending on wall colours: for dark walls $\mathrm{A}=0.9$, for grey and green ones $\mathrm{A}=0.7$, for light walls $\mathrm{A}=0.5$; I - intensity of solar radiation depending on time of day, latitude, season; $\alpha_{z}$ - convective heat-transfer coefficient on external walls surface $20 \mathrm{~W} /\left(\mathrm{m}^{2} \mathrm{~K}\right)$.

The nominal solar radiation intensity, called the solar constant, varies between 1.35 and $1.44 \mathrm{~kW} / \mathrm{m}^{2}$. This radiation during crossing the Earth's atmosphere is weakened. The measure of the weakening is the atmosphere transparency coefficient B. Its values depend on the season and the local atmosphere cleanness. Characteristic values $[8,11]$.

The sunlit area $F_{S N}$ is the area of the walls on which the sunrays fall directly and the roof one decreased by the area of windows on these walls.

$$
Q_{p n}=\sum_{\mathrm{i}} F_{S N i} \cdot k_{N_{i}}\left(T_{o b l}-T_{W}\right)
$$

Where the $I=1,2,3$ index means the wall through which heat is exchanged, taking the radiation into account. The sun is usually on the three walls of the warehouse.

Heat penetrating through windows $\mathrm{Q}_{\mathrm{ok}}$.

$$
Q_{o k}=F_{S Z} \cdot k_{S Z}\left(T_{o t}-T_{W}\right)
$$

Symbols in the equation (8) represent: $F_{S Z}$ - area of all window panels; $\mathrm{k}_{\mathrm{sz}}$ - heat transfer coefficient through window panels that depends on window types, window panels are single or double, glued multilayer. Detailed data about window panels give their manufacturers. The last component of the dependence is the heat penetrating into interior $Q_{o k p}$ in the path of radiation through window panels.

$$
Q_{o k n}=\left[F_{S Z N} \cdot I \cdot a \cdot\left(F_{S Z}-F_{S Z N}\right) I_{R}\right] \cdot b
$$

Symbols in the equation (9) represent: $F_{S Z N}$ - area of sunlit window panels; $F_{S Z}$ - total area of window panels; $I$ - solar radiation intensity; $I_{R}$ - scattered solar radiation intensity. $Q_{p b p}-$ heat penetrating through walls from buildings adjoining to warehouse (10):

$$
Q_{p b p}=\sum_{i} F_{p b p} \cdot k_{i}\left(T_{w m}-T_{w p}\right)
$$

Symbols in the equation (10) represent: $F_{p b p}$ - wall area; $k_{i}$ - wall penetrability; $T_{w m}$ - warehouse interior temperature; $T_{w p}$ - adjoining building's interior temperature. Saving of the energy consumption is sought in the roof suitable structure. Windows and skylights are located in the roof; these, light the interior in a natural way (thus reducing energy consumption for lighting). Pipe optical fibres are also used. The warehouse energy consumption is also affected by its location and alignment relative to the compass.

$Q_{\text {wen }}$ - heat lost or outflowing due to intended or unintended ventilation of interior. The temperature difference between the interior and environment as well as the pressure difference will decide unintended air exchange. Unintended air exchange is determined by the temperature difference between the interior and the environment as well as the pressure difference. The heat losses in this path are attempted to be decreased by reducing leaks and the use of various airlocks and air curtains.

The warehouse building leaktightness is understood as the ability to prevent air penetration through internal partitions. It regards, in particular, the air movement through joints of external partition elements. It is estimated that the air penetration is responsible for more than $30 \%$ of the costs borne for heating and ventilation LSM.

$Q_{\text {wew }}$ - heat of object ventilation is given by the dependence (11).

$$
Q_{w e n}=W \cdot c_{p}\left(T_{o t}-T_{W}\right)\left(1-\eta_{c}\right)
$$


Symbols in the equation (11) represent: $c_{p}$ - air specific heat; $T_{o t}-$ ambient temperature; $T_{W}$ - temperature inside warehouse; $W$ - quantity of air exchanged in a time unit between interior and environment.

The value of the exchange is influenced by the air streams enforced by the ventilation system due to hygienic or technical reasons, as well as the uncontrolled stream through technical holes, doors, gates, airlocks. The forced ventilation can be expressed by: the change rate that is the ratio of the exchanged air quantity to the interior volume $[1 / h]$ or $\left[\mathrm{m}^{3} / \mathrm{h}\right]$.

The warehouse ventilation system should be consistent with the standard PN-83/B-03430/Az3 that defines minimal values of ventilation air supplied to the defined rooms. The fresh air supply to rooms can significantly impact heat losses and that is why the EU regulations provide that the supply-exhaust ventilation of capacity higher than $500 \mathrm{~m}^{3} / \mathrm{h}$ must be equipped with an energy recovery system of efficiency exceeding $50 \%$. Since $1^{\text {st }}$ January 2016, in accordance with EU Commission regulation No. 1253/2014 of $7^{\text {th }}$ July 2014 on execution of European Parliament and Council Directive 2009/125/UC in relation to the eco-project requirements for ventilation systems (Journal of Laws EU L 337/8), the minimal thermal efficiency for supply-exhaust ventilation systems must be $\eta_{c}=67 \%$ and from January $2018-\eta_{c}=73 \%$.

$Q_{u r z}-$ heat emitted by devices operating in warehouse.

It regards forklift trucks and various types conveyors. They perform mechanical work the part of which impacts increasing potential energy $\Delta E_{p}$ of the goods stored on the warehouse racks. This part of the energy in the energy balance should be deducted from the work used by internal transport devices.

$$
\Delta E_{p}=\sum_{i} \dot{m}_{\iota} g h
$$

Symbols in the equation (12) represent: $m_{i}$ - total mass of goods placed on warehouse racks in considered time unit; $h$-storage height from floor level. From the point of view of a heat balance and energy consumption, the electric forklift trucks with electric drive are definitely more advantageous than the trucks with combustion drive and are at least $70 \%$ more efficient.

$Q_{0 \text { św }}$ - energy consumed by light sources

$$
Q_{\text {osw. }}=\sum Q_{\text {osw.jedn }}
$$

The correct luminous flux density is approx. $150 \mathrm{~lx} / \mathrm{m}^{2}$ - zone lighting control should be used if possible.

$Q_{\text {wew.wen }}$ - energy consumed for internal ventilation forced by fans.

Considering the temperature homogenization in the interior and the correct heat dissipation airflows and fans are used to equalize the temperature throughout the warehouse. The energy power consumed by the devices puts the air in motion, but finally, as a result of viscous friction of air particles, it is converted into the heat.

$Q_{0 \text { s }}-$ heat emitted by warehouse employees

$$
Q_{o s}=\sum Q_{j e d n}
$$

Every man working in a cold store emits heat. The heat is emitted through skin and the exhaled air; it is the so-called latent heat.

In the warehouse energy balance (dependence (1)) there are components strictly related to the construction technology of the warehouse wall, roof and floor. While testing the LSM energy consumption it can be noticed that the LSM energy consumption is 
significantly impacted by technical-organizational solutions. It is not justified to compare the energy consumption by warehouses of various intended use. The work character and primary goals may overrule energy consumption $[9,10]$.

\section{LSM energy consumption evaluation by coefficients}

The energy consumption by a warehouse is influenced by: goods turnover, assortment quantities, storage technology, warehouse equipment, work organization in a warehouse, degree of mechanization and automatization, advancement of automatic identification technics, microclimatic requirements, external factors: temperature and humidity, requirements inside warehouse, warehouse size.

Therefore, it seems reasonable to compare the energy consumption with warehouse units rotated in the considered time interval e.g. $\frac{k W h}{E P}$ in $\mathrm{kWh}$ per one palette or $24 \mathrm{~h}$. It is also possible to compare the global energy consumption with the warehouse floor area, its cubic volume or total quantity of warehouse nests on the rack storage. In the table 1 we present a comparison of exemplary evaluation indexes of LSM energy consumption that can be a measure of the warehouse energy consumption.

Table 1. Comparison of LSM energy consumption evaluation indexes. Own analysis.

\begin{tabular}{|c|c|c|}
\hline Item & Index & Formula \\
\hline 1 & $\begin{array}{l}\text { Global energy consumption per } \\
\text { warehouse unit }\end{array}$ & $W_{k j m}=\frac{\sum E_{i}}{k_{j m}}$ \\
\hline 2 & $\begin{array}{l}\text { Index of energy consumption per } \\
\text { warehouse area unit }\end{array}$ & $W_{F e}=\frac{\sum F_{i}}{F}$ \\
\hline 3 & $\begin{array}{l}\text { Index of energy consumption per } \\
\text { warehouse cubature unit }\end{array}$ & $W_{V}=\frac{\sum F_{i}}{V}$ \\
\hline 4 & $\begin{array}{l}\text { Index of energy consumption per rack } \\
\text { nest in time }\end{array}$ & $W_{g}=\frac{\sum F_{i}}{m}$ \\
\hline \multicolumn{3}{|c|}{$\begin{array}{l}\text { where: } k_{j m} \text { - number of warehouse units going through the warehouse in specific interval } \\
\mathrm{F}-\text { warehouse area; } \mathrm{V}-\text { volume (storage capacity) }\left[\mathrm{m}^{3}\right] ; \mathrm{m} \text { - number of rack nests; }\end{array}$} \\
\hline
\end{tabular}

\section{Directions of solutions minimizing energy consumption by unit warehouse}

While discussing minimization of energy consumption, we keep in mind the minimization of the indexes listed in table 1 . It does not concern the absolute values of the energy used by an individual warehouse, but it does concern the consumption comparison with the warehouse unit handled. The consumption will be the highest at low turnover in a big warehouse e.g. state reserves in cold stores. Low values will be in warehouses of quick turnover of a high throughput working in the cross-docking system.

The energy consumption minimization in case of cold stores can be obtained through: 1 . minimization of external impacts; 2 . minimization of internal heat sources; 3 . suitable solutions of warehouse internal infrastructure; 4. suitable warehouse and supporting system work organization; 5 . using green energy from own sources and energy recovery.

Ad. 1. The external impact means heat losses through walls and air exchange between the interior and external environment. The state of the situation can be improved through:

- the use of suitable heat insulating materials of economically justified quality and thickness, and avoiding the so-called thermal bridges; 
- the ventilation losses can be decreased by reducing controlled air flows, i.e. ensuring the tightness of buildings, traffic openings (doors, gates) surrounded by airlocks with insulating elements (sleeves, pneumatic shafts), openings protected with strip curtains or quick-rotating curtains - activated by proximity sensors. Finally, air curtains are being used - also automatically controlled. The air exchange should take place with as much control as possible through recuperation systems.

Ad. 2. The minimization of the energy supplied to the interior from internal sources regards the energy emitted by personnel, devices supporting warehouse work, lighting, and internal ventilation.

Ad. 3. Energy consumption in unit warehouses also depends on infrastructural solutions: flow racks, chutes, gravitational movement of goods. The transport devices should be equipped with energy recuperation systems, for example forklift trucks that operate intensively in an accelerating and braking cycle should have energy recovery systems. Possible benefits in this regard are presented in Fig. 2.
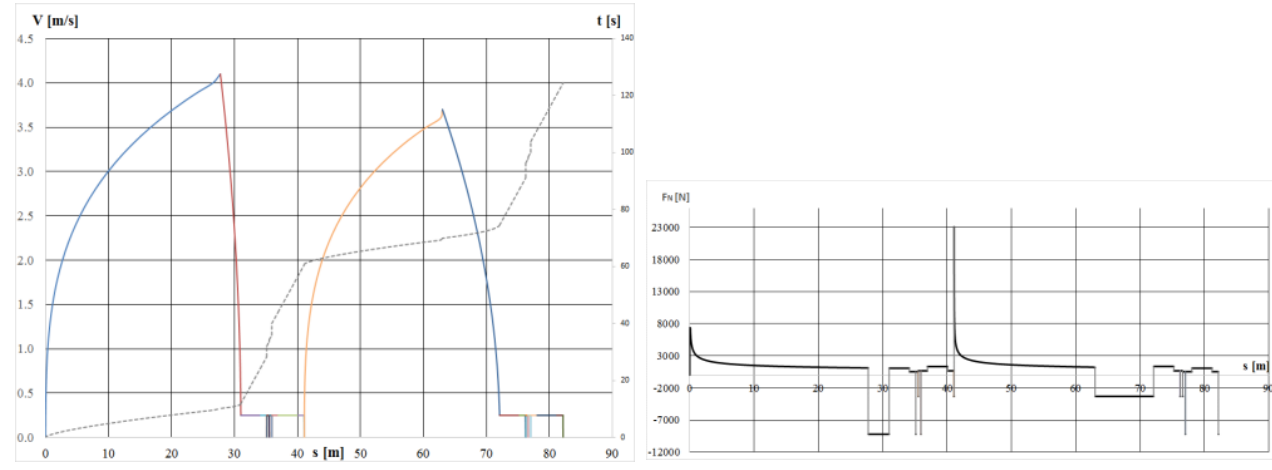

Fig. 2. The run of traffic changes in the function of distance and time of covering of particular movement phases. Own analysis.

Fig. 2 shows the speed profile of a forklift truck travel consistent with standard VDI2198 for Jungheinrich EFG-220 forklift truck so-called minimal time travel and the time of distance covering. Fig. $2 b$ shows run of driving forces and braking forces of the truck during operation in VDI2198 cycle. The fields on the graph between the force graph and the y-axis illustrate the energy consumed by the truck for drive and braking. It is possible, in this way, to calculate precisely the energy consumed by the truck and estimate potential benefits from the energy recovery during braking.

A warehouse full of goods is a big energy accumulator in a form of potential energy contained in the stored goods. That energy should be used. From this point of view, it is advantageous to situate the acceptance zone higher than the picking and release zones.

Ad. 4. Appropriate work organization, warehouse operation management and the arrangement of the goods has a huge impact on the energy consumption. For these purposes $\mathrm{ABC}$ and $\mathrm{XYZ}$ (analysis of the distribution of goods $\mathrm{ABC}$ : according to cumulated turnover, $\mathrm{XYZ}$ - according to the accuracy of the forecast) analyses are used. Picking and automatic identification enables considerable savings regarding work and time. The systems of picking processes support: pick-by-light, pick-by-voice. Depending on the warehouse operation character, savings can bring a dispersed system of goods storage in various zones making picking possible for many employees at the same time. Undoubtedly the automated picking systems can be also more advantageous than hand picking. 
Ad. 5. The energy supplying the warehouse, ensuring its functioning, should be used as efficiently as possible e.g. by using heat pumps in the cooling and heating systems. Large areas of the roofs (flat areas) of the warehouses are used for the arrangement of solar cells. Only in such a situation can the warehouse, under certain circumstances, achieve zero energy condition in an annual balance.

\section{Summary}

The goal of this analysis was to identify all essential factors affecting energy consumption in a warehouse. Formally speaking, it is expressed by the energy consumption equation (1), and quantitative trends can be shown by the indexes proposed in Table 1. In many cases, it is possible to shape the warehouse and its equipment in such a way, that the energy consumption, equals zero e.g. in an annual scale. It must be noted that energy consumption in a warehouse is not always the most important goal. The superior goal is always the efficient handling of the flow of goods. In many cases we may agree to extensive automation, often energy-consuming, instead of short handling time and correct picking. In various situations, however, it is worth taking into account the energy consumption of a warehouse facility.

\section{References}

1. P. Zajac, Evaluation method of energy consumption in logistic warehouse systems (Springer, DOI 10.1007/978-3-319-22044-4, 2015)

2. P. Zajac, Springer Proceedings in Energy, (Springer, 195-200, DOI: 10.1007/978-3319-16901-9)

3. P. Zajac, The energy consumption in refrigerated warehouses (Springer, DOI 10.1007/978-3-319-40898-9, 2016)

4. P. Zajac, Advances in Intelligent Systems and Computing (AISC) 350, 345-355, DOI: 10.1007/978-3-319-15796-2_35 (2015)

5. P. Keskiewicz, B. Sniechowska, J. Danielewicz, Instal 2, 27-30 (2012)

6. J. Danielewicz, K. Golecki, A guide for the designer of the boiler house (Publishing House of the Wrocław University of Technology, Wroclaw, 2004)

7. J. Danielewicz, et al., Energy, 108 (2016)

8. P. Zajac, Archives of Civil and Mechanical Engineering 11, 2, 479-492 (2011)

9. J. Grajnert, S. Kwasniowski, Heat transfer dynamics in refrigerated vehicles, Refrigeration - Polish Engineering Association (NOT) 33, 37-39 (1998)

10. S. Jebaraj, S. Iniyan, Renewable and Sustainable Energy Reviews 10, 4, 281-311 (2006)

11. E.-R. Schramek, Taschenbuch fur Heinzung und Klimatechnik, (07/08, Herman Recknagel, Industrieverlag-Munchen, 2010) 